\section{TATRA \\ MOUNTaiNS \\ Mathematical Publications}

DOI: $10.2478 / \mathrm{tmmp}-2021-0027$

Tatra Mt. Math. Publ. 79 (2021), 199-212

\title{
EXISTENCE AND MULTIPLICITY \\ OF POSITIVE SOLUTIONS FOR A THIRD-ORDER TWO-POINT BOUNDARY VALUE PROBLEM
}

\author{
SERGEY SMIRNOV \\ University of Latvia, Riga, LATVIA
}

\begin{abstract}
We study the existence and multiplicity of positive solutions for a third-order two-point boundary value problem by applying Krasnosel'skii's fixed point theorem. To illustrate the applicability of the obtained results, we consider some examples.
\end{abstract}

\section{Introduction}

We study a two-point boundary value problem consisting of the nonlinear third-order differential equation

$$
x^{\prime \prime \prime}=a(t) f(x), \quad 0<t<1,
$$

and the boundary conditions

$$
x(0)=0, \quad x(1)=0, \quad x^{\prime}(1)=0 .
$$

We assume throughout that $f:[0, \infty) \rightarrow[0, \infty)$ is continuous, $a:[0,1] \rightarrow[0, \infty)$ is continuous and does not vanish identically on any subinterval of $[0,1]$.

The purpose of the paper is to give results on the existence and multiplicity of positive solutions to (1), (2) by applying Krasnosel'skii's fixed point theorem. By a positive solution of (1), (2) we understand $C^{3}[0,1]$ function which is positive on $0<t<1$ and satisfies differential equation (11) for $0<t<1$ and boundary conditions (2). However, note that if $f(0)=0$, then boundary value problem (1), (2) always has the trivial solution. Since $f(x)$ is not defined for $x<0$,

(C) 2021 Mathematical Institute, Slovak Academy of Sciences. 2010 Mathematics Subject Classification: 34B15, $34 \mathrm{~B} 18$.

Keywords: Nonlinear boundary value problems, existence and multiplicity of positive solutions, Green's function, Krasnosel'skii's fixed point theorem .

(c) (1) ()- Licensed under the Creative Commons BY-NC-ND 4.0 International Public License. 
every solution of (11), (2) is nonnegative. We will show in the sequel that every nonnegative nontrivial solution of (1), (2) is positive.

In fact, our main results state that for each given positive integer $n$, we can indicate $f$ so that problem (11), (2) has at least $n$ positive solutions. To obtain these results, we first rewrite problem (11), (2) as an equivalent integral equation by constructing the corresponding Green's function. Then, we apply Krasnosel'skii's fixed point theorem in a cone [11,16].

Much research has been done on two-point boundary value problems for third-order differential equations in the last decades. As recent contributions, we cite the papers of Cabada and Dimitrov [4, Gritsans and Sadyrbaev [10], Kelevedjiev and Todorov [15. Krasnosel'skii's fixed point theorem in a cone has been widely used to study the existence and multiplicity of positive solutions of second and higher-order boundary value problems. One of the early works on the subject was a paper by Guo and Lakshmikantham [12. Further research in this direction was carried out by Erbe and Wang [5], Henderson and Thompson [13], Anderson and Davis [2], Baxley and Haywood [3], Graef, Qian and Yang [6], Graef and Yang [7] 9], Zhao, Wang and Ge [20. For survey of known results and additional references we refer the reader to the monograph by Agarwal, O'Regan and Wong [1]. A great contribution to the development of the subject was made by Webb and Infante [14, 17, 19]. The author would like to highlight the paper by Graef and Yang [7, which motivated the present investigation. We prove the existence of multiple positive solutions for our main problem in the same manner as in [7], but we use another splitting of the cone.

Since our main tool in this paper is Krasnosel'skii's fixed point theorem, let us state this theorem for the reader's convenience.

Theorem 1.1 (Krasnosel'skii, 16]). Let $E$ be a Banach space and $K \subset E$ be a cone in $E$. Assume $\Omega_{1}$ and $\Omega_{2}$ are open subsets of $E$ with $0 \in \Omega_{1}$ and $\bar{\Omega}_{1} \subset \Omega_{2}$, $T: K \cap\left(\bar{\Omega}_{2} \backslash \Omega_{1}\right) \rightarrow K$ is a completely continuous operator such that:

$$
\begin{array}{ccc}
\|T x\| \leq\|x\|, \forall x \in K \cap \partial \Omega_{1} \quad \text { and } & \|T x\| \geq\|x\|, \forall x \in K \cap \partial \Omega_{2}, \quad \text { or } \\
\|T x\| \geq\|x\|, \forall x \in K \cap \partial \Omega_{1} \quad \text { and } \quad\|T x\| \leq\|x\|, \forall x \in K \cap \partial \Omega_{2} .
\end{array}
$$

Then $T$ has a fixed point in $K \cap\left(\bar{\Omega}_{2} \backslash \Omega_{1}\right)$.

The paper contains four sections besides the Introduction. In Section 2, we rewrite the main problem as an equivalent integral equation, by constructing the corresponding Green's function. Also, we give some inequalities for the Green's function here. In Section 3, we consider the existence of at least one positive solution for the problem. Section 4 is devoted to the existence of multiple positive solutions for the problem. Finally, we provide some examples to illustrate our results in Section 5. 


\section{Construction and estimation of the Green's function}

Our first goal is to rewrite problem (11), (2) as an equivalent integral equation. Therefore, let us consider the linear equation

$$
x^{\prime \prime \prime}=h(t), 0<t<1,
$$

together with boundary conditions (2).

Proposition 2.1. If $h:[0,1] \rightarrow \mathbb{R}$ is a continuous function, then boundary value problem (3), (2) has a unique solution

$$
\begin{aligned}
x(t)= & \int_{0}^{t}\left[\frac{1}{2} s^{2}(1-t)^{2}\right] h(s) d s \\
& +\int_{t}^{1}\left[\frac{1}{2}(1-s) t((s-t)+(1-t) s)\right] h(s) d s,
\end{aligned}
$$

that we can rewrite as

$$
x(t)=\int_{0}^{1} G(t, s) h(s) d s,
$$

where

$$
G(t, s)=\frac{1}{2} \begin{cases}s^{2}(1-t)^{2}, & 0 \leq s \leq t \leq 1 \\ (1-s) t((s-t)+(1-t) s), & 0 \leq t \leq s \leq 1\end{cases}
$$

Pr o of. To prove the proposition we use the variation of parameters formula

$$
x(t)=c_{1}+c_{2} t+c_{3} t^{2}+\frac{1}{2} \int_{0}^{t}(s-t)^{2} h(s) d s .
$$

In view of boundary conditions (2), we get $c_{1}=0$ and

$$
\begin{aligned}
& c_{2}=-\int_{0}^{1}(s-1)^{2} h(s) d s-\int_{0}^{1}(s-1) h(s) d s, \\
& c_{3}=\frac{1}{2} \int_{0}^{1}(s-1)^{2} h(s) d s+\int_{0}^{1}(s-1) h(s) d s .
\end{aligned}
$$


Thus, we have

$$
\begin{aligned}
& \text { Thus, we have } \begin{aligned}
x(t)= & \left(t^{2}-t\right) \int_{0}^{1}(s-1) h(s) d s+\left(\frac{1}{2} t^{2}-t\right) \int_{0}^{1}(s-1)^{2} h(s) d s \\
& +\frac{1}{2} \int_{0}^{t}(s-t)^{2} h(s) d s \\
= & \int_{0}^{t}\left[\frac{1}{2} s^{2}(1-t)^{2}\right] h(s) d s+\int_{t}^{1}\left[\frac{1}{2}(1-s) t((s-t)+(1-t) s)\right] h(s) d s .
\end{aligned}
\end{aligned}
$$

The uniqueness follows from the fact, that the homogeneous problem $x^{\prime \prime \prime}=0$, (2) has only the trivial solution.

Hence boundary value problem (10), (2) is equivalent to the integral equation

$$
x(t)=\int_{0}^{1} G(t, s) a(s) f(x(s)) d s, \quad 0 \leq t \leq 1,
$$

in the sense that $x$ is a solution of (1), (2) if and only if it is a solution of (5). Here $G(t, s)$ denotes the Green's function for the problem $x^{\prime \prime \prime}=0$, (2), and is explicitly given by (4).

In order to prove the existence of positive solutions, we also need some inequalities for the Green's function $G(t, s)$.

Proposition 2.2. For all $(t, s) \in[0,1] \times[0,1]$, we have

$$
0 \leq G(t, s) \leq \frac{s^{2}(1-s)}{2(1+s)} \text {. }
$$

If $(t, s) \in(0,1) \times(0,1)$, then

$$
G(t, s)>0 \text {. }
$$

P r o of. The first part of inequality (6) and inequality (7) is obvious. Let us find the maximum of $G(t, s)$ for each $s$ with respect to $t$.

For $0 \leq s \leq t \leq 1$, the maximum occurs at

$$
t=s \quad \text { and is equal to } \frac{s^{2}(1-s)^{2}}{2} .
$$

If $0 \leq t \leq s \leq 1$, the maximum occurs at

$$
t=\frac{s}{1+s} \text { and is equal to } \frac{s^{2}(1-s)}{2(1+s)} .
$$

Since for all $(t, s) \in[0,1] \times[0,1]$,

$$
\frac{s^{2}(1-s)^{2}}{2}=\frac{s^{2}(1-s)(1-s)(1+s)}{2(1+s)}=\frac{s^{2}(1-s)\left(1-s^{2}\right)}{2(1+s)} \leq \frac{s^{2}(1-s)}{2(1+s)},
$$

we get the proof.

Note that inequality (6) is sharp. 
Proposition 2.3. For all $(t, s) \in[1 / 14,1 / 2] \times[0,1]$, we have

$$
G(t, s) \geq \frac{1}{4} \cdot \frac{s^{2}(1-s)}{2(1+s)}
$$

Pr o o f. Consider

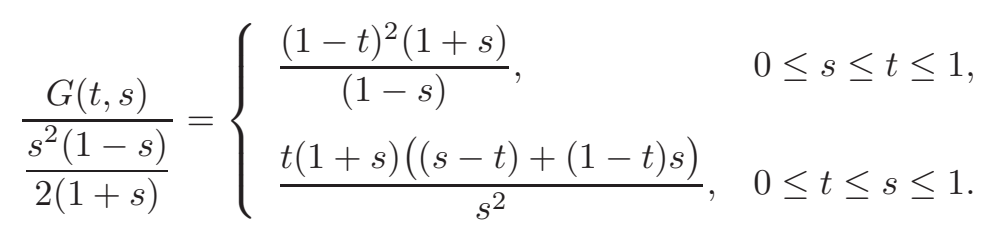

Further, for $\Lambda_{1}=\{(t, s): 1 / 14 \leq t \leq 1 / 2,0 \leq s \leq 1, s \leq t\}$, we have

$$
\min _{\Lambda_{1}} \frac{G(t, s)}{\frac{s^{2}(1-s)}{2(1+s)}}=\min _{\Lambda_{1}} \frac{(1-t)^{2}(1+s)}{(1-s)}=\frac{1}{4} .
$$

If $\Lambda_{2}=\{(t, s): 1 / 14 \leq t \leq 1 / 2,0 \leq s \leq 1, t \leq s\}$, then

$$
\min _{\Lambda_{2}} \frac{G(t, s)}{\frac{s^{2}(1-s)}{2(1+s)}}=\min _{\Lambda_{2}} \frac{t(1+s)((s-t)+(1-t) s)}{s^{2}}=\frac{13}{49} .
$$

Therefore,

$$
\frac{G(t, s)}{\frac{s^{2}(1-s)}{2(1+s)}} \geq \frac{1}{4} \quad \text { for } \quad \frac{1}{14} \leq t \leq \frac{1}{2}, \quad 0 \leq s \leq 1 .
$$

Proposition 2.4. Every nonnegative nontrivial solution $x(t)$ of (11), (2) is positive.

Proof. Suppose that there exists $t_{0} \in(0,1)$ such that $x\left(t_{0}\right)=0$. Since boundary value problem (11), (2) is equivalent to integral equation (5) we get

$$
x\left(t_{0}\right)=\int_{0}^{1} G\left(t_{0}, s\right) a(s) f(x(s)) d s=0 .
$$

Since $G\left(t_{0}, s\right) a(s) f(x(s)) \geq 0$ for all $s \in[0,1]$, then

$$
G\left(t_{0}, s\right) a(s) f(x(s))=0 \quad \text { for all } s \in[0,1] .
$$

Since $G\left(t_{0}, s\right)>0$ for all $s \in(0,1)$ we get that $x^{\prime \prime \prime}(s)=a(s) f(x(s))=0$ for all $s \in(0,1)$. Therefore, $x(s)$ is a polynomial of degree at most two. Since $x(s)$ satisfies boundary conditions (2) it follows that $x(s)=0$ for all $s \in[0,1]$. We get the contradiction. 


\section{Existence of positive solutions}

In this section, we study the existence of positive solutions for boundary value problem (11), (2). Throughout, we shall use the following notations:

$$
\begin{gathered}
f_{0}=\lim _{x \rightarrow 0+} \frac{f(x)}{x}, \quad f_{\infty}=\lim _{x \rightarrow+\infty} \frac{f(x)}{x} \\
I_{1}=\left(\int_{0}^{1} \frac{s^{2}(1-s)}{2(1+s)} a(s) d s\right)^{-1}<\left(\max _{0 \leq t \leq 1} \int_{1 / 14}^{1 / 2} G(t, s) a(s) d s\right)^{-1}=I_{2} .
\end{gathered}
$$

For our constructions, consider the Banach space $C[0,1]$ with the norm

Define a cone $K$ in $C[0,1]$ by

$$
\|x\|=\max _{0 \leq t \leq 1}|x(t)|, x \in C[0,1] .
$$

$$
K=\left\{x \in C[0,1] \mid x(t) \geq 0, \min _{\frac{1}{14} \leq t \leq \frac{1}{2}} x(t) \geq \frac{1}{4}\|x\|\right\},
$$

and an integral operator $T: K \rightarrow C[0,1]$ by

$$
(T x)(t)=\int_{0}^{1} G(t, s) a(s) f(x(s)) d s, \quad 0 \leq t \leq 1 .
$$

It is easy to see that boundary value problem (11), (2) has a solution $x$ if and only if $x$ is a fixed point of the operator $T$. Also, it is well-known that $T: K \rightarrow C[0,1]$ is a completely continuous operator.

Proposition 3.1. $T(K) \subset K$.

Pro of. From inequality (6), it follows that for $x \in K,(T x)(t) \geq 0$ on $[0,1]$. Also, for $x \in K$, we have from (6) that

so that

$$
(T x)(t)=\int_{0}^{1} G(t, s) a(s) f(x(s)) d s \leq \int_{0}^{1} \frac{s^{2}(1-s)}{2(1+s)} a(s) f(x(s)) d s,
$$

$$
\|T x\| \leq \int_{0}^{1} \frac{s^{2}(1-s)}{2(1+s)} a(s) f(x(s)) d s .
$$

And next, if $x \in K$, we have by (8) and (9),

$$
\begin{aligned}
\min _{\frac{1}{14} \leq t \leq \frac{1}{2}}(T x)(t) & =\min _{\frac{1}{14} \leq t \leq \frac{1}{2}} \int_{0}^{1} G(t, s) a(s) f(x(s)) d s \\
& \geq \frac{1}{4} \int_{0}^{1} \frac{s^{2}(1-s)}{2(1+s)} a(s) f(x(s)) d s \geq \frac{1}{4}\|T x\| .
\end{aligned}
$$




\section{EXISTENCE AND MULTIPLICITY OF POSITIVE SOLUTIONS}

Theorem 3.2. If $f_{0}<I_{1}$ and $f_{\infty}>4 I_{2}$ (particularly $f_{0}=0$ and $f_{\infty}=\infty$ -superlinear case), then boundary value problem (1), (2) has at least one positive solution.

Pr o of. Since $f_{0}<I_{1}$, we may choose $r_{1}>0$ such that $f(x) \leq I_{1} x$ for $0<x \leq r_{1}$. Thus, if $x \in K$ and $\|x\|=r_{1}$, then

$$
\begin{aligned}
(T x)(t) & =\int_{0}^{1} G(t, s) a(s) f(x(s)) d s \leq \int_{0}^{1} \frac{s^{2}(1-s)}{2(1+s)} a(s) f(x(s)) d s \\
& \leq I_{1}\|x\| \int_{0}^{1} \frac{s^{2}(1-s)}{2(1+s)} a(s) d s=\|x\|, \quad 0 \leq t \leq 1 .
\end{aligned}
$$

Now, if we let $\Omega_{1}=\left\{x \in C[0,1] \mid\|x\|<r_{1}\right\}$, then $\|T x\| \leq\|x\|$ for $x \in K \cap \partial \Omega_{1}$.

On the other hand, since $f_{\infty}>4 I_{2}$, there exists $\widehat{r}_{2}>0$ such that $f(x) \geq 4 I_{2} x$ for $x \geq \widehat{r}_{2}$. Let $r_{2}=\max \left\{2 r_{1}, 4 \widehat{r}_{2}\right\}$ and $\Omega_{2}=\left\{x \in C[0,1]\|\| x \|<r_{2}\right\}$. Then $x \in K$ and $\|x\|=r_{2}$ implies

$$
\min _{\frac{1}{14} \leq t \leq \frac{1}{2}} x(t) \geq \frac{1}{4}\|x\|=\frac{1}{4} r_{2} \geq \widehat{r}_{2} .
$$

Thus, if $s \in[1 / 14,1 / 2]$, then $x(s) \in\left[\widehat{r}_{2}, r_{2}\right]$, and we get

$$
\begin{aligned}
\|T x\| & =\max _{0 \leq t \leq 1} \int_{0}^{1} G(t, s) a(s) f(x(s)) d s \geq \max _{0 \leq t \leq 1} \int_{1 / 14}^{1 / 2} G(t, s) a(s) f(x(s)) d s \\
& \geq 4 I_{2} \max _{0 \leq t \leq 1} \int_{1 / 14}^{1 / 2} G(t, s) a(s) x(s) d s \\
& \geq 4 I_{2} \frac{1}{4}\|x\| \max _{0 \leq t \leq 1} \int_{1 / 14}^{1 / 2} G(t, s) a(s) d s=\|x\| .
\end{aligned}
$$

Hence, $\|T x\| \geq\|x\|$ for $x \in K \cap \partial \Omega_{2}$.

Therefore, from Theorem 1.1, it follows that $T$ has a fixed point in $K \cap$ $\left(\bar{\Omega}_{2} \backslash \Omega_{1}\right)$. Since the set $K \cap\left(\bar{\Omega}_{2} \backslash \Omega_{1}\right)$ does not contain zero element (trivial solution), it follows that this fixed point yields a positive solution of (10), (2).

Theorem 3.3. If $f_{0}>4 I_{2}$ and $f_{\infty}<I_{1}$ (particularly $f_{0}=\infty$ and $f_{\infty}=0$ -sublinear case), then boundary value problem (10), (2) has at least one positive solution. 
Proof. Since $f_{0}>4 I_{2}$, we may choose $r_{1}>0$ such that $f(x) \geq 4 I_{2} x$ for $0<x \leq r_{1}$. Then, for $x \in K$ and $\|x\|=r_{1}$, we have

$$
\begin{aligned}
\|T x\| & =\max _{0 \leq t \leq 1} \int_{0}^{1} G(t, s) a(s) f(x(s)) d s \geq \max _{0 \leq t \leq 1} \int_{1 / 14}^{1 / 2} G(t, s) a(s) f(x(s)) d s \\
& \geq 4 I_{2} \max _{0 \leq t \leq 1} \int_{1 / 14}^{1 / 2} G(t, s) a(s) x(s) d s \\
& \geq 4 I_{2} \frac{1}{4}\|x\| \max _{0 \leq t \leq 1} \int_{1 / 14}^{1 / 2} G(t, s) a(s) d s=\|x\| .
\end{aligned}
$$

Thus, we may let $\Omega_{1}=\left\{x \in C[0,1] \mid\|x\|<r_{1}\right\}$ so that $\|T x\| \geq\|x\|$ for $x \in$ $K \cap \partial \Omega_{1}$.

Now, since $f_{\infty}<I_{1}$, there exists $\widehat{r}_{2}>0$ so that $f(x) \leq I_{1} x$ for $x \geq \widehat{r}_{2}$.

Case (a): Suppose $f$ is bounded, say $f(x) \leq N$ for all $x \in(0, \infty)$. In this case choose $r_{2}=\max \left\{2 r_{1}, N I_{1}^{-1}\right\}$ so that for $x \in K$ with $\|x\|=r_{2}$ we have

$$
(T x)(t)=\int_{0}^{1} G(t, s) a(s) f(x(s)) d s \leq N \int_{0}^{1} \frac{s^{2}(1-s)}{2(1+s)} a(s) d s \leq r_{2}
$$

and therefore, we get $\|T x\| \leq\|x\|$.

Case (b): If $f$ is unbounded, we can choose $r_{2}>\max \left\{2 r_{1}, \widehat{r}_{2}\right\}$ such that $f(x) \leq f\left(r_{2}\right)$ for $0<x \leq r_{2}$. Then for $x \in K$ and $\|x\|=r_{2}$ we have

$$
\begin{aligned}
(T x)(t) & =\int_{0}^{1} G(t, s) a(s) f(x(s)) d s \leq \int_{0}^{1} \frac{s^{2}(1-s)}{2(1+s)} a(s) f(x(s)) d s \\
& \leq \int_{0}^{1} \frac{s^{2}(1-s)}{2(1+s)} a(s) f\left(r_{2}\right) d s \leq I_{1} r_{2} \int_{0}^{1} \frac{s^{2}(1-s)}{2(1+s)} a(s) d s=r_{2} \\
& =\|x\|, \quad 0 \leq t \leq 1 .
\end{aligned}
$$

Therefore, in both cases, we may put $\Omega_{2}=\left\{x \in C[0,1] \mid\|x\|<r_{2}\right\}$ and for $x \in K \cap \partial \Omega_{2}$ we have $\|T x\| \leq\|x\|$. Thus, from Theorem[1.1] it follows that $T$ has a fixed point in $K \cap\left(\bar{\Omega}_{2} \backslash \Omega_{1}\right)$. Since the set $K \cap\left(\bar{\Omega}_{2} \backslash \Omega_{1}\right)$ does not contain zero element (trivial solution), it follows that this fixed point yields a positive solution of (1), (2). 


\section{EXISTENCE AND MULTIPLICITY OF POSITIVE SOLUTIONS}

THEOREM 3.4. If there exist constants $0<r_{1}<r_{2}$ such that $f(x) \leq I_{1} r_{1}$ for $x \in\left[0, r_{1}\right]$ and $f(x) \geq I_{2} r_{2}$ for $x \in\left[r_{2} / 4, r_{2}\right]$, then boundary value problem (11), (2) has at least one positive solution $x(t)$ such that $r_{1} \leq\|x\| \leq r_{2}$.

Proof. If $x \in K$ and $\|x\|=r_{1}$, then

$$
\begin{aligned}
(T x)(t) & =\int_{0}^{1} G(t, s) a(s) f(x(s)) d s \leq \int_{0}^{1} \frac{s^{2}(1-s)}{2(1+s)} a(s) f(x(s)) d s \\
& \leq I_{1} r_{1} \int_{0}^{1} \frac{s^{2}(1-s)}{2(1+s)} a(s) d s=r_{1}=\|x\|, \quad 0 \leq t \leq 1 .
\end{aligned}
$$

If we let $\Omega_{1}=\left\{x \in C[0,1] \mid\|x\|<r_{1}\right\}$, then $\|T x\| \leq\|x\|$ for $x \in K \cap \partial \Omega_{1}$.

If $x \in K$ with $\|x\|=r_{2}$, then for every $s \in[1 / 14,1 / 2]$ we have

$$
\min _{\frac{1}{14} \leq s \leq \frac{1}{2}} x(s) \geq \frac{1}{4}\|x\|=\frac{1}{4} r_{2} \quad \text { and } \quad x(s) \in\left[r_{2} / 4, r_{2}\right] \text {. }
$$

Therefore,

$$
\begin{aligned}
\|T x\| & =\max _{0 \leq t \leq 1} \int_{0}^{1} G(t, s) a(s) f(x(s)) d s \geq \max _{0 \leq t \leq 1} \int_{1 / 14}^{1 / 2} G(t, s) a(s) f(x(s)) d s \\
& \geq I_{2} r_{2} \max _{0 \leq t \leq 1} \int_{1 / 14}^{1 / 2} G(t, s) a(s) d s=r_{2}=\|x\| .
\end{aligned}
$$

Thus, we may let $\Omega_{2}=\left\{x \in C[0,1]\|\| x \|<r_{2}\right\}$ so that $\|T x\| \geq\|x\|$ for $x \in K \cap \partial \Omega_{2}$.

Therefore, from Theorem 1.1] it follows that $T$ has a fixed point in $K \cap$ $\left(\bar{\Omega}_{2} \backslash \Omega_{1}\right)$. Since the set $K \cap\left(\bar{\Omega}_{2} \backslash \Omega_{1}\right)$ does not contain zero element (trivial solution), it follows that this fixed point yields a positive solution of (11), (2).

\section{Existence of multiple positive solutions}

The following two propositions will be used in the proof of our main result of this section.

Proposition 4.1. Suppose that there exists $r>0$ such that $f(x) \leq I_{1} \frac{4}{5} r$ for $x \in[0, r]$. If $x \in K$ with $\|x\|=\rho$, where $\frac{4}{5} r \leq \rho \leq r$, then $\|T x\| \leq \rho$. 


\section{SERGEY SMIRNOV}

Proof. If $x \in K$ with $\|x\|=\rho$, then for $t \in[0,1]$ we have

$$
\begin{aligned}
(T x)(t) & =\int_{0}^{1} G(t, s) a(s) f(x(s)) d s \leq \int_{0}^{1} \frac{s^{2}(1-s)}{2(1+s)} a(s) f(x(s)) d s \\
& \leq I_{1} \frac{4}{5} r \int_{0}^{1} \frac{s^{2}(1-s)}{2(1+s)} a(s) d s=\frac{4}{5} r \leq \rho, \quad \text { or } \quad\|T x\| \leq \rho .
\end{aligned}
$$

Proposition 4.2. Suppose that there exists $r>0$ such that $f(x) \geq I_{2} r$ for $x \in[r / 5, r]$. If $x \in K$ with $\|x\|=\rho$, where $\frac{4}{5} r \leq \rho \leq r$, then $\|T x\| \geq \rho$.

Pr o of. If $x \in K$ with $\|x\|=\rho$, where $\frac{4}{5} r \leq \rho \leq r$, then for every $s \in[1 / 14,1 / 2]$ we have

$$
\min _{\frac{1}{14} \leq s \leq \frac{1}{2}} x(s) \geq \frac{1}{4}\|x\|=\frac{1}{4} \rho \quad \text { and } \quad x(s) \in[\rho / 4, \rho] \quad \text { or } \quad x(s) \in[r / 5, r] .
$$

Therefore,

$$
\begin{aligned}
\|T x\| & =\max _{0 \leq t \leq 1} \int_{0}^{1} G(t, s) a(s) f(x(s)) d s \geq \max _{0 \leq t \leq 1} \int_{1 / 14}^{1 / 2} G(t, s) a(s) f(x(s)) d s \\
& \geq I_{2} r \max _{0 \leq t \leq 1} \int_{1 / 14}^{1 / 2} G(t, s) a(s) d s=r \geq \rho .
\end{aligned}
$$

Now, we are ready to give the main result of this section. Let:

- $\lfloor\alpha\rfloor=\max \{k \in \mathbb{Z} \mid k \leq \alpha\}$ be the floor of a real number $\alpha$ and

- $\lceil\alpha\rceil=\min \{k \in \mathbb{Z} \mid k \geq \alpha\}$ be the ceiling of $\alpha$.

Theorem 4.3. Suppose that there exist constants $0<r_{1}<r_{2}<\cdots<r_{n}(n \geq 3)$. If $f(x) \leq I_{1} \frac{4}{5} r_{2 i-1}$ for $x \in\left[0, r_{2 i-1}\right], 1 \leq i \leq\left\lceil\frac{n}{2}\right\rceil$ and $f(x) \geq I_{2} r_{2 j}$ for $x \in\left[r_{2 j} / 5, r_{2 j}\right], 1 \leq j \leq\left\lfloor\frac{n}{2}\right\rfloor$, then boundary value problem (11), (12) has at least $(n-1)$ positive solutions $x_{k}(t)$ such that

$$
r_{k} \leq\left\|x_{k}\right\|<r_{k+1}, \quad 1 \leq k \leq(n-1) .
$$

Proof. Let $l_{m} \in\left(4 r_{m} / 5, r_{m}\right)$ and $l_{m}>r_{m-1}, 2 \leq m \leq n$. Define

and

$$
\Omega_{m}^{l_{m}}=\left\{x \in C[0,1] \mid\|x\|<l_{m}\right\}, \quad 2 \leq m \leq n,
$$

$$
\Omega_{s}^{r_{s}}=\left\{x \in C[0,1] \mid\|x\|<r_{s}\right\}, \quad 1 \leq s \leq n .
$$




\section{EXISTENCE AND MULTIPLICITY OF POSITIVE SOLUTIONS}

Then, from Proposition 4.1 and Proposition 4.2, we have:

$$
\begin{aligned}
\|T x\| \leq\|x\| \quad \text { for } \quad x \in K \cap \partial \Omega_{2 p-1}^{l_{2 p-1},} & 2 \leq p \leq\left\lceil\frac{n}{2}\right\rceil, \\
\text { and } \quad \text { for } \quad x \in K \cap \partial \Omega_{2 i-1}^{r_{2 i-1}}, & 1 \leq i \leq\left\lceil\frac{n}{2}\right\rceil ; \\
\|T x\| \geq\|x\| \quad \text { for } \quad x \in K \cap \partial \Omega_{2 j}^{l_{2 j}}, & 1 \leq j \leq\left\lfloor\frac{n}{2}\right\rfloor, \\
\text { and } \quad \text { for } \quad x \in K \cap \partial \Omega_{2 j}^{r_{2 i}}, & 1 \leq j \leq\left\lfloor\frac{n}{2}\right\rfloor .
\end{aligned}
$$

From Theorem [1.1, we see that $T$ has fixed point in each of the sets $K \cap$ $\left(\bar{\Omega}_{m}^{l_{m}} \backslash \Omega_{m-1}^{r_{m-1}}\right), 2 \leq m \leq n$. Thus, boundary value problem (11), (2) has at least $(n-1)$ positive solutions.

\section{Examples}

In this section, we consider some examples to illustrate the applicability of the results established in Section 3 and Section 4.

ExAmple. Consider boundary value problem consisting of the differential equation

$$
x^{\prime \prime \prime}=100 \frac{x+5 x^{3}}{3+x^{2}}, \quad 0<t<1,
$$

and boundary conditions (2). We have

$$
a(t)=100, \quad f(x)=\frac{x+5 x^{3}}{3+x^{2}},
$$

and therefore, $f_{0}=\frac{1}{3}, f_{\infty}=5, I_{1} \approx 0.3776,4 I_{2} \approx 4.405$. Since $f_{0}<I_{1}$ and $f_{\infty}>4 I_{2}$, by Theorem 3.2, boundary value problem (10), (2) has at least one positive solution, which is depicted in Figure 11. The initial conditions for this solution are:

$$
x(0)=0, \quad x^{\prime}(0) \approx 6.8852, \quad x^{\prime \prime}(0) \approx-30.9744 .
$$

EXAMPLE. Consider boundary value problem for the differential equation

$$
x^{\prime \prime \prime}=100 t\left(\frac{3 x}{5}+\frac{12 x}{1+x^{2}}\right), \quad 0<t<1,
$$

with boundary conditions (2). Here

$$
a(t)=100 t, \quad f(x)=\frac{3 x}{5}+\frac{12 x}{1+x^{2}},
$$

and therefore, $f_{0}=12.6, f_{\infty}=0.6, I_{1} \approx 0.6585,4 I_{2} \approx 12.253$. Since $f_{0}>4 I_{2}$ and $f_{\infty}<I_{1}$, by Theorem 3.3 , boundary value problem (11), (2) has at least one 
positive solution, which is depicted in Figure 2. The initial conditions for this solution are $x(0)=0, x^{\prime}(0) \approx 47.2081, x^{\prime \prime}(0) \approx-143.215$.

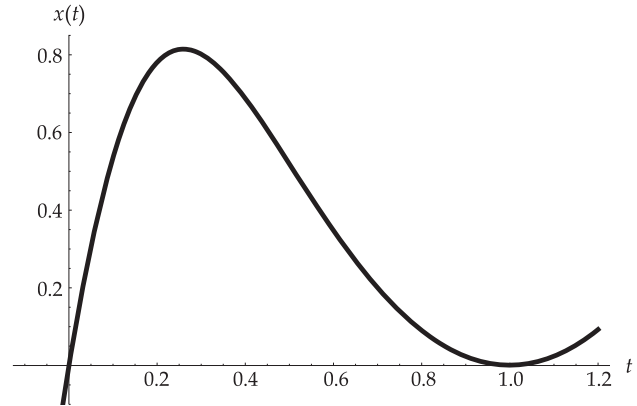

FIGURE 1.

Solution of (10), (2).

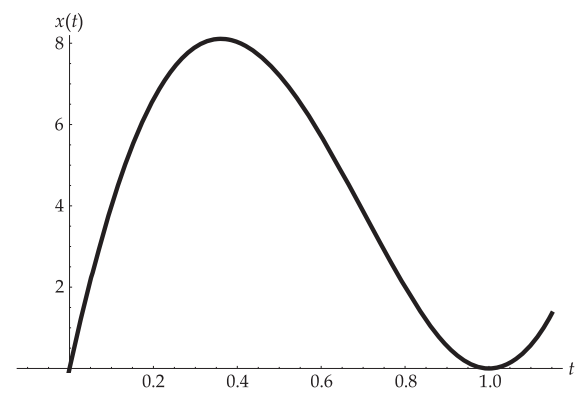

Figure 2.

Solution of (11), (2).

ExAmPLE. Consider boundary value problem for the differential equation

$$
x^{\prime \prime \prime}=10 x^{2} e^{6-x}, \quad 0<t<1,
$$

with boundary conditions (2). We have:

$$
a(t)=10, \quad f(x)=x^{2} e^{6-x}, \quad \frac{4}{5} I_{1} \approx 3.021, \quad I_{2} \approx 11.013
$$

If we choose $r_{1}=0.006, r_{2}=5, r_{3}=73$, we get

$$
\begin{aligned}
& f(x) \leq I_{1} \frac{4}{5} r_{1} \quad \text { for } \quad x \in\left[0, r_{1}\right], \\
& f(x) \leq I_{1} \frac{4}{5} r_{3} \quad \text { for } \quad x \in\left[0, r_{3}\right], \\
& f(x) \geq I_{2} r_{2} \quad \text { for } \quad x \in\left[r_{2} / 5, r_{2}\right] .
\end{aligned}
$$

Therefore, by Theorem (4.3), boundary value problem (12), (2) has at least two positive solutions $x_{1}(t)$ and $x_{2}(t)$ such that

$$
0.006 \leq\left\|x_{1}\right\|<5 \leq\left\|x_{2}\right\|<73 .
$$

Solutions $x_{1}(t)$ and $x_{2}(t)$ are depicted in Figure 3 and Figure 4 . The initial conditions for these solutions are $x_{1}(0)=0, x_{1}^{\prime}(0) \approx 0.2618675, x_{1}^{\prime \prime}(0) \approx-1.189665$ and $x_{2}(0)=0, x_{2}^{\prime}(0) \approx 57.148, x_{2}^{\prime \prime}(0) \approx-297.923$. 


\section{EXISTENCE AND MULTIPLICITY OF POSITIVE SOLUTIONS}

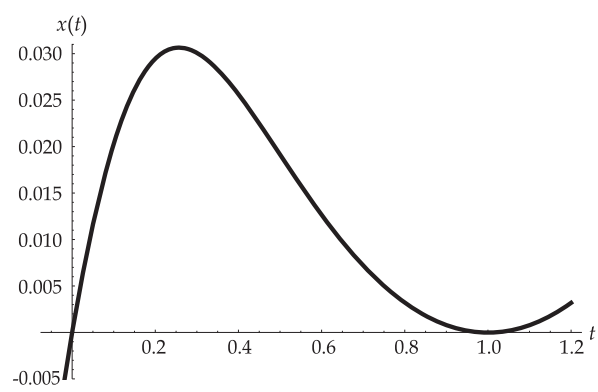

FiguRE 3.

Solution $x_{1}$ of (12), (2).

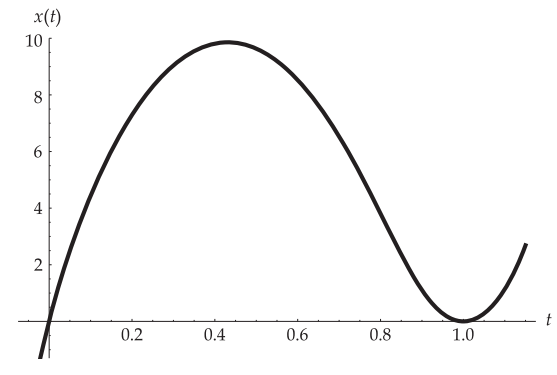

Figure 4.

Solution $x_{2}$ of (12), (2).

Acknowledgement. The author wishes to express his thanks to the referee for very valuable suggestions and careful corrections.

\section{REFERENCES}

[1] AGARWAL, R.P.-O'REGAn, D.-WOnG, P. J. Y.: Positive Solutions of Differential, Difference and Integral Equations. Kluwer Academic, Dordrecht, 1998.

[2] ANDERSON, D.R.-DAVIS, J.M.: Multiple solutions and eigenvalues for third-order right focal boundary value problem, J. Math. Anal. Appl. 267 (2002), 135-157.

[3] BAXLEY, J,-HAYWOOD, L. J.: Multiple positive solutions of nonlinear boundary value problems, Dyn. Contin. Discrete Impuls. Syst. Ser. A Math. Anal. 10 (2003), 157-168.

[4] CABADA, A.-DIMITROV, ND: Third-order differential equations with three-point boundary conditions, Open Mathematics 19 (2021), 11-31.

[5] ERBE, L.H.-WANG, H.: On the existence of positive solutions of ordinary differential equations, Proc. Amer. Math. Soc. 120 (1994), 743-748.

[6] GRAEF, J.R.-QIAN, C.-YANG, B.: A three point boundary value problem for nonlinear fourth order differential equations, J. Math. Anal. Appl. 287 (2003), 217-233.

[7] GRAEF, J.R.-YANG, B.: Multiple positive solutions to a three point third order boundary value problem, Discrete Contin. Dyn. Syst. (2005), suppl., 337-344.

[8] GRAEF, J.R.-YANG, B.: Positive solutions of a third order nonlocal boundary value problem, Discrete Contin. Dyn. Syst. Ser. S 1 (2008), 89-97.

[9] GRAEF, J.R.-YANG, B.: Existence and nonexistence of positive solutions of a nonlinear third order boundary value problem, Electron. J. Qual. Theory Differ. Equ. 9 (2008), 1-13.

[10] GRITSANS, A.-SADYRBAEV, F.: A two-point boundary value problem for third order asymptotically linear systems, Electron. J. Qual. Theory Differ. Equ. 28 (2019), 1-24.

[11] GUO, D.-LAKSHMIKANTHAM, V.: Nonlinear Problems in Abstract Cones. Academic Press, San Diego, 1988. 
[12] GUO, D.-LAKSHMIKANTHAM, V.: Multiple solutions of two-point boundary value problems of ordinary differential equations in Banach spaces, J. Math. Anal. Appl. 129 (1988), 211-222.

[13] HENDERSON, J.-THOMPSON, H. B.: Multiple symmetric positive solutions for a second order boundary value problem, Proc. Amer. Math. Soc. 128 (2000), 2373-2379.

[14] INFANTE, G.-WEBB, J.R. L.: Positive solutions of some nonlocal boundary value problems, Abstract and Applied Analysis. 18 (2003), 1047-1060.

[15] KELEVEDJIEV, P. S.-TODOROV, T. Z.: Existence of solutions of nonlinear third-order two-point boundary value problems, Electron. J. Qual. Theory Differ. Equ. 23 (2019), 1-15.

[16] KRASNOSEL'SKII, M. A.: Positive Solutions of Operator Equations. Noordhoff, Groningen, 1964.

[17] WEBB, J.R.L. : Optimal constants in a nonlocal boundary value problem, Nonlinear Anal. 63 (2005), 672-685.

[18] WEBB, J.R. L.-INFANTE, G.: Positive solutions of nonlocal boundary value problems involving integral conditions, Nonlinear Differential Equations and Appl. (NoDEA) 15 (2008), 45-67.

[19] WEBB, J.R. L.-INFANTE, G.: Nonlocal boundary value problems of arbitrary order, Journal of the London Mathematical Society 79 (2009), no. 1, 238-258.

[20] ZHAO, J.-WANG, P.- GE, W.: Existence and nonexistence of positive solutions for a class of third order BVP with integral boundary conditions in Banach spaces, Commun. Nonlinear Sci. Numer. Simul. 16 (2011), 402-413.

Received (September 24, 20221

\author{
Sergey Smirnov \\ Institute of Mathematics and \\ Computer Science \\ University of Latvia \\ Raina bulvaris 29 \\ LV-1459 Riga \\ LATVIA
}

Faculty of Physics, Mathematics and

Optometry

University of Latvia

Jelgavas iela 3

LV-1004 Riga

LATVIA

E-mail: srgsm@inbox.lv 